\title{
A Philosophical Treatise and Educational Aspects on Fuzzy Control
}

\author{
MICHAEL GR. VOSKOGLOU \\ School of Enginnering \\ University of Peloponnese \\ Patras, GREECE \\ mvoskoglou@gmail.com
}

\begin{abstract}
Fuzzy Logic has found nowadays many applications to almost all sectors of human activity, with fuzzy control being one of the most important such applications. A control system regulates the behavior of a device or another system with the help of a feedback controller. A fuzzy control system is a control system that analyses the input data in terms of variables which take continuous values in the interval [0, 1]. The present article studies in detail the operation of fuzzy control systems. In the effort of presenting our results in the simplest possible way, so that they could be understood even by a non-expert, we have chosen a geometrical approach based on an example with a building's central heating controller.
\end{abstract}

Keywords: fuzzy set (FS); fuzzy logic (FL); control system (CS); triangular fuzzy number (TFN); center of gravity (COG) defuzzification technique; fuzzy control system (FCS); fuzzy approximation theorem (FAT); fuzzy associative memory (FAM).

Received: June 15, 2020. Revised: November 4, 2020, Accepted: November 19, 2020. Published: November 30, 2020.

\section{Introduction}

There is no doubt that the enormous progress of science and technology owe a lot to the Aristotle's (384-322 BC) bivalent logic, which dominated for centuries the human way of thinking and civilization. However, even from the time of Buddha (around 500 BC), Heraclitus (535-475 BC) and Plato (427-377 BC) views have appeared too discussing the existence of a third area between "true" and "false", where these two opposites can exist together. More recent philosophers like Hegel, Marx, Engels, Russel and others supported and cultivated further those ideas, but the first integrated propositions of multivalued logics appeared only during the $20^{\text {th }}$ century by Jan Lukasiewicz (1858-1956) and Alfred Tarski (1901-1983) (for more details see Voskoglou, 2019, Section 2). Max Black (1937) introduced the concept of vague set being a premonition of the Zadeh's fuzzy set (FS) (Zadeh, 1965), which led to the development of the infinitevalued in the interval $[0,1]$ fuzzy logic (FL) (Zadeh, 1973).

But, while Zadeh was trying to spread out the message of fuzziness, he received many tough critiques for his radical ideas from three different directions (Kosko, 1993). The first direction came from many scientists asked for some practical applications. In fact, such applications started to appear in industry during the 1970's, the first one being in the area of cement kiln control (Umbers \& King, 1980). This is an operation demanding the control of a highly complex set of chemical interactions by dynamically managing 40-50 "rules of thumb". This was followed by E. H. Mamdani's (1975) work in the Queen Mary College of London, who designed the first fuzzy system for controlling a steam engine and later the operation of traffic lights. Another type of fuzzy inference systems was developed later in Japan by Takagi-SugenoKang (Sugeno, 1985). It is well known that nowadays FSs and FL have found many and important applications to almost all sectors of human activity.
The second direction is related to the probability theorists, who claimed that FL cannot do any more than probability does. Membership degrees, taking values in the same with probabilities interval $[0,1]$, are actually hidden probabilities, fuzziness is a kind of disguised randomness, and the multivalued logic is not a new idea. It took a long time to become universally understood, just recently, that fuzziness does not oppose probability, but actually supports and completes it by treating successfully the cases of the existing in the real world uncertainty which is caused by reasons different from randomness (Kosko, 1990). The expressions "John's membership degree in the FS of clever people is 0.7 " and "The probability of John to be clever is 0.7 ", although they look similar, they actually have essentially different meanings. The former means that John is a rather clever person, whereas the latter means that John, according to the principle of the excluded middle, is either clever or not, but his outlines (heredity, academic studies, etc.) suggests that the probability to be clever is high $(70 \%)$.

The third direction comes from bivalent logic. Many of its traditional supporters, based on a culture of centuries, argue that, since this logic works effectively in science, functions the computers and explains satisfactorily the phenomena of the real world, except perhaps those that happen in the boundaries, there is no reason to make things more complicated by introducing the unstable principles of a multivalued logic.

FL, however, aims exactly at smoothing the situation in the boundaries! Look, for example, at the graph of Fig. 1 corresponding to the FS T of "tall people". People with heights less than $1.50 \mathrm{~m}$ are considered of having membership degree 0 in $T$. The membership degree is continuously increasing for heights greater than $1.50 \mathrm{~m}$, taking its maximal value 1 for heights equal or greater than $1.80 \mathrm{~m}$. Therefore, the "fuzzy part" of the graph - which is conventionally represented in Fig. 1 by the straight line segment AC, but its exact form depends upon the way in which the membership function has been defined - lies in the area of the rectangle $\mathrm{ABCD}$ defined by the $\mathrm{OX}$ axis, its 
parallel through the point $\mathrm{E}$ and the two perpendicular to it lines at the points $\mathrm{A}$ and $\mathrm{B}$.

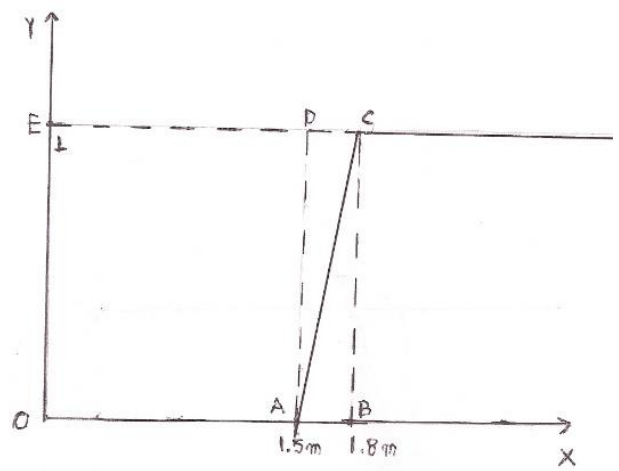

Fig. 1. The fuzzy set of "tall people"

In fact, the way of perceiving a concept (e.g. "tall") is different from person to person, depending on the "signals" that each one receives from the real world about it. Mathematically speaking, that means that the definition of the membership function of a FS is not unique, depending on the observer's personal criteria. The only restriction is that this definition must be compatible to the common logic, because otherwise the corresponding FS does not give a reliable description of the corresponding real situation. On the contrary, bivalent logic defines a bound, e.g. $1.80 \mathrm{~m}$, above which people are considered to be tall and under which are considered to be short. Consequently, one with height $1.79 \mathrm{~m}$ is considered to be short, whether another with height $1.81 \mathrm{~m}$ is tall!

There are also strong voices of anger against FL, without bothering to present any logical arguments about it. Those voices, characterize FL as the tool for making the science unstable, or more emphatically as the "cocaine of science"! Such voices, however, appear frequently in analogous cases of the history of science and must be simply ignored.

Zadeh realized that FSs are connected to words (adjectives and adverbs) of the natural language; e.g. the adjective "tall" indicates the FS of the tall people, since "how tall is everyone" is a matter of degree. A grammatical sentence may contain many adjectives and/or adverbs, therefore it correlates a number of FSs. A synthesis of grammatical sentences, i.e. a group of FSs related to each other, forms what we call a fuzzy system. A fuzzy system provides empirical advices, mnemonic rules and common logic in general. It is not only able to use its own knowledge to represent and explain the real world, but can also increase it with the help of the new data; in other words, it learns from the experience. This is actually the way in which humans think. Nowadays, a fuzzy system can control, for example, the function of an electric washing-machine or send signals for purchasing shares from the stock exchange, etc.

The target of the present paper is the study of the fuzzy control systems, which are the most important category of fuzzy systems. The rest of the paper is organized as follows: Section II studies the structure of the traditional control systems of bivalent logic. Section III is devoted to the theoretical study of fuzzy control, which is highly based on the fuzzy approximation theorem. The structure of a fuzzy control system is fully illustrated by the example of controlling a building's central heating boiler presented in Section IV and the article closes with the general conclusions, which are stated in Section V.

\section{Control Systems}

The notion of a system has a very broad context in general. It can be defined as a set of interacting components forming an integrated whole. We distinguish among physical, social, economic, biological, abstract (mathematical, philosophical, etc.) systems and many others.

The study of a system is usually performed through the modelling process. A model is a simplified representation of the basic characteristics of a real system, which identifies and simplifies the relationships among those characteristics in a form amenable to analysis. Several kinds of models are used according to the form of the corresponding problem, their most common types being the mathematical and simulation models. In the former case mathematical symbols and methods are used for representing the system. In the latter case the system is represented by a sequence of logical orders, usually functioning with the help of computers (for more details see Taha 1967, Chapter 1).

A control system (CS) regulates the behaviour of other devices or systems by using a feedback controller. We distinguish between two main types of CSs, the open-loop and the closed-loop CSs. In the former case the action of the controller is independent of the process variable $(P V)$ to be controlled. This is, for example, the case of a central heating boiler controlled by a timer switching on or off the boiler after a certain period of time, regardless the building's temperature (which is the PV). In the latter case, on the contrary, the controller's action depends on the PV and the desired value of it, which is usually referred as the set point $(S P)$. More explicitly, the difference SP-VP, called the $S P-V P$ error, is applied as a control signal on the purpose of equalizing the values of SP and VP. In case of the boiler analogy, for example, this is achieved with the help of a thermostat, which, working as a sensor, monitors the building's temperature to ensure that the controller's output maintains it close to the temperature set by the thermostat (SP). The structure of a closed-loop CS is graphically represented in Fig. 2, retrieved from the Web.

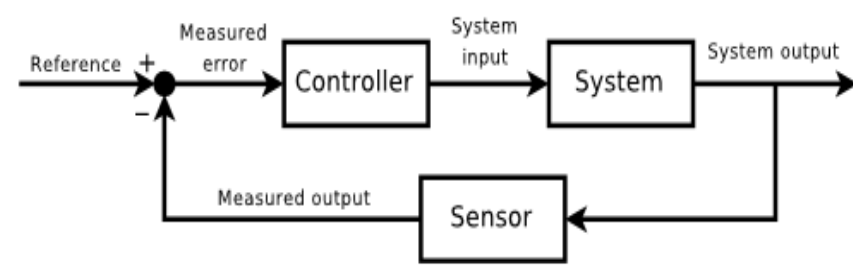

Fig. 2. Structure of a closed-loop control system

The operation of a controller is regulated by a set of $I F$ THEN logical rules. A rule connects a process or event A to another process or event B. For example, IF the temperature of the building is lower than $18^{\circ} \mathrm{C}(\mathrm{A})$, then the thermostat sends a signal, which, with the help of one or more mechanical valves, turns on the boiler (B). On the contrary, if the building's temperature exceeds $27^{\circ} \mathrm{C}(\mathrm{A})$, then the thermostat sends a signal to the controller to turn off the boiler (B). Knowledge, therefore, could be seen as a combination of such rules, an idea originated at least from the Aristotle's time. 


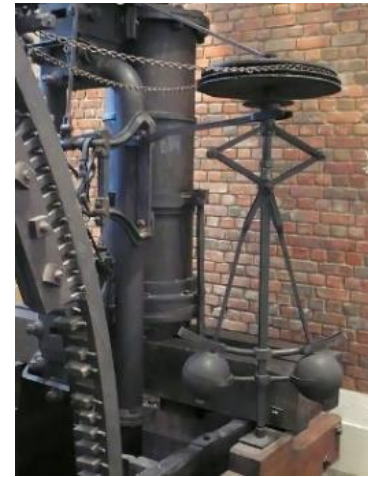

Fig. 3. Centrifugal governor in a Boulton and Watt engine of 1788

CSs are used to achieve, through automatic operation, increased productivity, high quality products and improved performance of a device or another system. Control theory dates from 1868, when J. C. Maxwell described the theoretical basis for the operation of the governors (Fig. 3), i.e. mechanical devices for regulating the operation of industrial engines. The traditional CSs are described nowadays with mathematical models using one or more differential equations that define the CS's response to its inputs. Such CSs, initiated by N. Minorsky in 1922, are usually referred as PID (proportional-integral-derivative) controllers. Although the main application of mathematical control theory is the design of CSs for industry (CSs engineering), other important applications can also be found in life sciences, computer engineering, sociology and operation research (portal.dnb.de, 2020).

\section{Fuzzy Control}

In many cases the mathematical model of a classical controller may not exist, or may be too "expensive" in terms of computer processing power and memory. The classical expert systems of Artificial Intelligence (AI) (e.g. see Voskoglou \& Salem, 2020), for example, work by using 100 - 1000 bivalent IF-THEN rules. It has been estimated, however, that the human intelligence could be approached by a machine using at least $10^{5}$ such rules! In such cases, therefore, a system based on empirical rules could be more effective.

This was a good reason to introduce principles of FL for constructing "clever" CSs. It is of worth noting that, although in many cases genetic algorithms and neural networks can perform as well as FL, the latter has the advantage of expressing the solution of the problems in the natural language. In this way, the human experience can be used for designing the controller, which makes easier the mechanization of tasks that have been already performed successfully by humans. FL needs also logical rules but, in contrast to bivalent logic, does not need too many rules.

A fuzzy control system (FCS) is a CS that analyzes the input values in terms of variables which take on continuous values in the interval $[0,1]$. On the contrary the traditional CSs operate on the basis of the discrete values 0 (false) and 1 (true) only. The structure of a FCS, which is graphically represented in Fig. 4, retrieved from the Web, is relatively simple and similar to the structure of a traditional CS (Fig. 2).

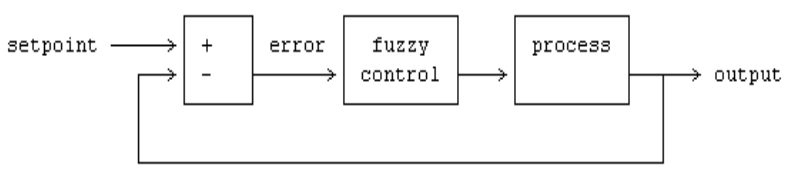

Fig. 4. Structure of a fuzzy control system

The interest on FCSs sparked by the development of a system for accelerating, breaking and stopping the vehicles of the Sedai Subway, a rapid transit network in Japan. S. Yasunobu and S. Miyamoto of Hitachi provided in 1985 simulations that demonstrated the feasibility of FCSs in that case. Since then, a wide range of FCSs was developed by Japanese engineers concerning vacuum cleaners, refrigerators, air conditions, autofocusing cameras, voice controlled helicopters, robots, etc. In 1988 Japan established the Laboratory of Intelligent Fuzzy Engineering (LIFE), a cooperative of 48 companies, on the purpose of pursuing applied fuzzy research. Development of FCSs is also proceeding in USA and Europe, though in a smaller scale than in Japan. NASA, the US Environmental Protection Agency, big firms as Boeing, General Motors, Chrysler, Whirpool, etc., have worked on FL for improved automatic transmissions, energy efficient electric motors and several other applications.

An important advantage of the FCSs with respect to the traditional CSs is that they do not need the existence of a model for studying the corresponding real world system. FCS design is an empirical approach, basically based on the trialand-error method characterized by repeated attempts, which are continued until success or until the subject stops trying (Athanassopoulos \& Voskoglou, 2020, Section 3). The general process of designing a FCS involves the following steps:

- Determine the system's operational specifications, inputs and outputs.

- Choose the FSs for the inputs and outputs.

- Document the set of IF-THEN fuzzy rules.

- Choose the suitable defuzzification method.

- Run tests to validate the system's behavior.

- Complete document by adjusting details as required and release to production.

An IF-THEN fuzzy rule is of the form: If $\mathrm{X}$ is $\mathrm{A}$, then $\mathrm{Y}$ is $\mathrm{B}$, where A, B are FS on the crisp sets of the input $\mathrm{X}$ and the output $Y$ respectively. For example: If the temperature $(\mathrm{X})$ is very low $(\mathrm{A})$, then the boiler $(\mathrm{Y})$ works in full rate $(\mathrm{B})$.

The operation of a FCS is based on the Fuzzy Approximation Theorem) (FAT) (Kosko, 1992). Roughly speaking, the FAT theorem, named so because the abbreviation "FAT theorem" sounds better than "FA theorem", states that any system can always be "covered" or approximated by a fuzzy system with a finite number of IFTHEN fuzzy rules and is geometrically represented in Fig. 5 (Kosko, 1993).

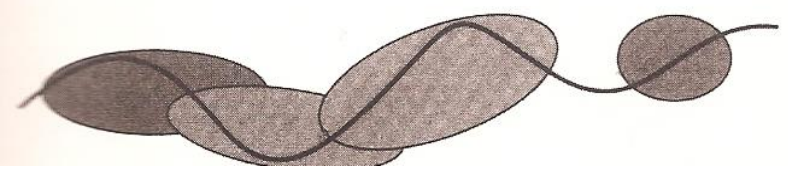

Fig. 5. The FAT theorem 
The existing in Fig. 5 curve represents the original system and each of the patches covering it corresponds to an IFTHEN fuzzy rule. The covering fuzzy system is the set of all those fuzzy rules, i.e. the set of all patches. The more uncertain an IF-THEN rule, the bigger the corresponding patch. If a rule is too accurate, then is not fuzzy and the corresponding patch degenerates to a point on the curve. More knowledge means more rules, i.e. more patches and better covering. The better the curve is covered by the patches, the cleverer the covering fuzzy system.

The design of a FCS that has been highlighted above will be fully illustrated by the example that follows in the next section. For more details about the theory and applications of Fuzzy Controllers the reader may look at Iqbal, 2012, Michels et al., 2006, Tanaka and Wang, 2001, Reznik, 1997, etc.

\section{An Application}

\subsection{Triangular Fuzzy Numbers}

For the better understating of the present section the reader is assumed to be familiar with the basics of the theory of Fuzzy Numbers (FNs) and in particular of Triangular FNs (TFNs), being the simplest form of FNs (Kaufmann \& Gupta, 1991). Here it is simply recalled that a TFN $A=(a, b, c)$, with a, b and c real numbers such that $\mathrm{a}<\mathrm{b}<\mathrm{c}$, is a FS on the set $\boldsymbol{R}$ of real numbers with membership function

$$
y=m(x)= \begin{cases}\frac{x-a}{b-a}, & x \in[a, b] \\ \frac{c-x}{c-b}, & x \in[b, c] \\ 0, & x<a \text { or } x>c\end{cases}
$$

The graph of the TFN $A=(a, b, c)$ is the triangle $A B C$ of Fig. 6. It is straightforward to check that the center of gravity (COG) of this triangle, i.e. the intersection point $\mathrm{G}$ of its medians, has coordinates $(\mathrm{x}, \mathrm{y})=\left(\frac{\mathrm{a}+\mathrm{b}+\mathrm{c}}{3}, \frac{1}{3}\right)$ (Voskoglou, 2019, Section 5).

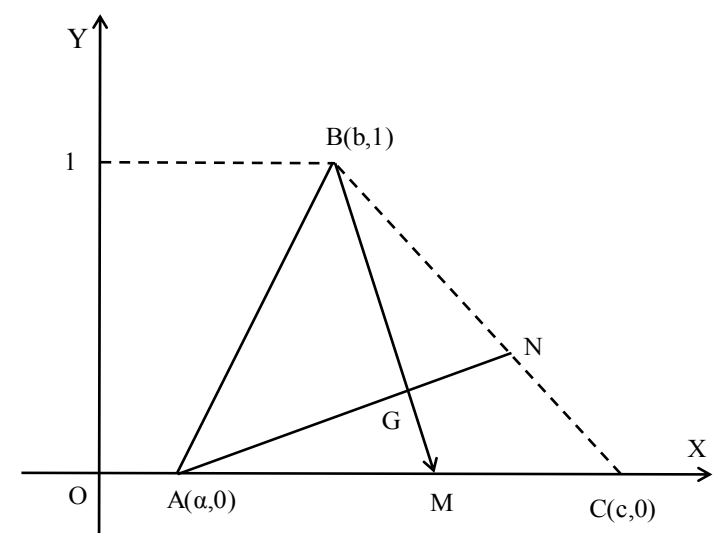

Fig. 6. Graph and COG of the TFN (a, b, c)

According to the COG defuzzification technique (Voskoglou, 2019, Section 4 ) the x-coordinate of G can be used as a ranking function $\mathrm{R}$ for defufuzzifying the TFNs. Therefore, we can write

$$
\mathrm{R}(\mathrm{A})=\frac{\mathrm{a}+\mathrm{b}+\mathrm{c}}{3}
$$

There are several other defuzzification approaches in use, the most commonly used being the "height" method, which takes the value of the biggest contributor (greatest output value).

\subsection{Fuzzy Control of a Central Heating Boiler}

Reconsider the case of controlling a building's central heating boiler, where the input $\mathrm{X}$ is the building's temperature in degrees of Celsius and the output $\mathrm{Y}$ is the rate of the boiler's operation expressed in a climax from 0 to 100 .

Without loss of generality and for reasons of simplicity let us consider only three FSs on $\mathrm{X}$, namely $\mathrm{A}_{1}=$ low temperature $\left(\leq 18^{0} \mathrm{C}\right), \mathrm{A}_{2}=$ normal temperature (between $15^{0}$ $\mathrm{C}$ and $\left.25^{\circ} \mathrm{C}\right)$ and $\mathrm{A}_{3}=$ high temperature $\left(>22^{\circ} \mathrm{C}\right)$. Assume also that the corresponding FSs on $\mathrm{Y}$ are $\mathrm{B}_{1}=$ high rate of the boiler's operation $(\geq 70), B_{2}=$ low rate of the boiler's operation (between 70 and 20) and $\mathrm{B}_{3}=$ boiler's turn off (rate between 30 and 0 ).

The simplest way of representing geometrically the FSs defined above is by using triangles (TFNs), although one could use trapezoids (trapezoidal FNs) as well, or any other figure representing the membership functions of the defined FSs. Using triangles, the $\mathrm{A}_{\mathrm{i}}$ 's are represented on the coordinate system XOY and the Bi's on the coordinate system $\mathrm{X}^{\prime} \mathrm{O}^{\prime} \mathrm{Y}^{\prime}$ of Fig. 7. The triangles representing the FSs $A_{1}$ and $A_{2}$ are not complete, because we could have temperatures lower than $0^{0} \mathrm{C}$ or higher than $27^{0} \mathrm{C}$. Those triangles can, however, be always completed in case of temperatures being outside the bounds of Fig. 7.

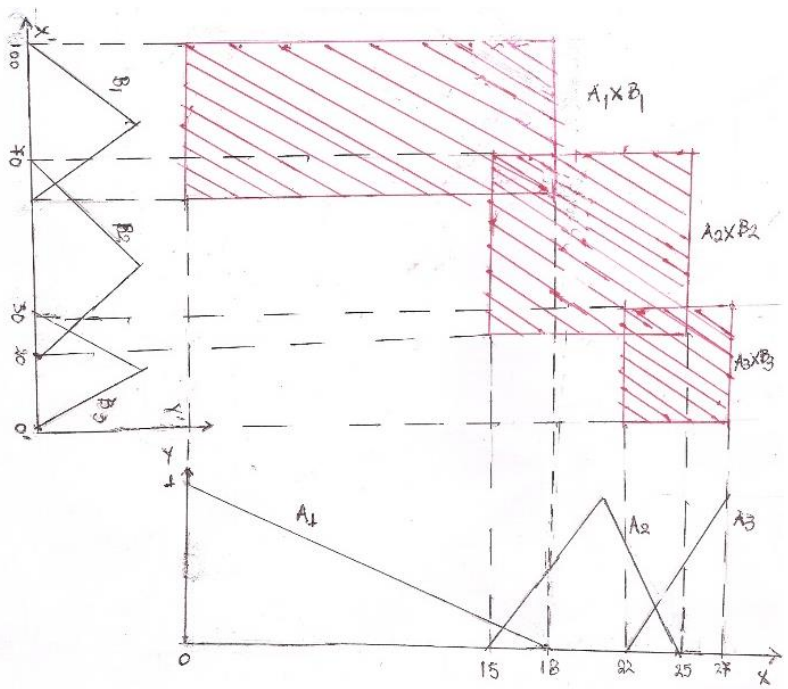

Fig. 7. The fuzzy control system of the boiler

In Fig. 7, the perpendicular lines to the $\mathrm{OX}$ and $\mathrm{O}^{\prime} \mathrm{X}$ ' axes from the vertices of the triangles $A_{i}$ and $B_{i}$ respectively lying on those axes define the rectangles $A_{i} \times B_{i}, i=1,2,3$. In this way the system obtains the required FAT, with the rectangles being the patches of the following fuzzy rules:

- $\mathrm{A}_{1} \times \mathrm{B}_{1}$ : IF the building's temperature is low, THEN the boiler operates in full rate. 
- $\mathrm{A}_{2} \times \mathrm{B}_{2}$ : IF the building's temperature is normal, THEN the boiler operates in low rate.

- $\mathrm{A}_{3} \times \mathrm{B}_{3}$ : IF the building's temperature is high, THEN the boiler turns off.

Those three patches cover a curve which represents the traditional control system of the boiler. That curve starts at the top of the patches on the left and ends at the bottom on the right. In case of a dynamic system changing by the time, the corresponding curve is moving. However, if the curve's movement is not too fast, the patches can be extended or moved in order to cover always the curve. In other words a fuzzy system has usually the ability to cover or approximate an existing dynamic system.

The boiler, however does not "understand" the fuzzy rules. In order to respond one must give to it a number instead, representing the rate of its operation. This is succeeded by defuzzifying the corresponding FSs. Assume, for example, that the building's temperature is $19^{0} \mathrm{C}$. This temperature has membership degrees zero in the FSs $\mathrm{A}_{1}$ and $A_{3}$ on $X$ and nonzero in $A_{2}$. This means that the fuzzy rule $A_{2}$ $x B_{2}$ will be fully activated $(100 \%)$. Since the triangle $B_{2}$ represents the $\mathrm{TFN} \mathrm{B}_{2}(20,45,70)$, using the $\mathrm{COG}$ defuzzification technique one finds by equation (1) that $\mathrm{R}\left(\mathrm{B}_{2}\right)=45$, i.e. the boiler receives the order to operate in a low rate.

Assume now that the building's temperature drops to $16^{0}$ $\mathrm{C}$, which has membership degrees zero in $\mathrm{A}_{3}$ and nonzero in $A_{1}$ and $A_{2}$ (Fig. 7). In this case, therefore, both the rules $A_{2} X$ $\mathrm{B}_{2}$ and $\mathrm{A}_{1} \times \mathrm{B}_{1}$ will be activated. In Fig. 8, BAC and HOZ correspond to the triangles $A_{2}$ and $A_{1}$ of Fig. 7 respectively, while the point $\mathrm{E}$ corresponds to the temperature of $16^{0} \mathrm{C}$.

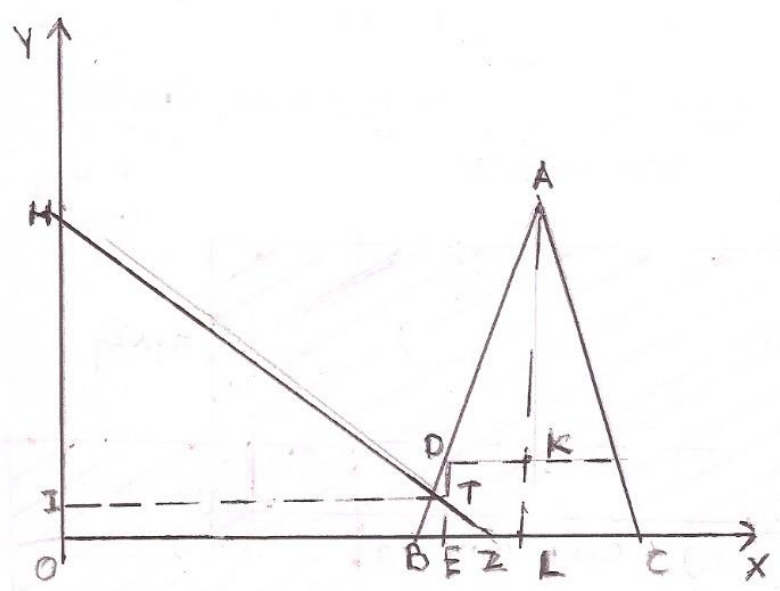

Fig. 8. The triangles $A_{1}$ and $A_{2}$ (inputs) of Fig. 7

Applying Thales' theorem in the triangle ALB one finds that $\frac{\mathrm{AB}}{\mathrm{DB}}=\frac{\mathrm{AL}}{\mathrm{KL}}$ and $\frac{\mathrm{BA}}{\mathrm{BD}}=\frac{\mathrm{BL}}{\mathrm{BE}}=\frac{5}{1}$, which gives that $\mathrm{KL}=$ $\frac{1}{5} \mathrm{AL}=\frac{1}{5}=\mathrm{DE}$. Therefore, $(\mathrm{DEB})=\frac{1}{10}$. We also have that $(\mathrm{BAC})=5 \quad$ and $(\mathrm{ADEC})=(\mathrm{BAC})-(\mathrm{DEB})=5-\frac{1}{10}=\frac{49}{10}$, which gives that $\frac{(\mathrm{ADEC})}{(\mathrm{ABC})}=\frac{49}{50}=0.98$. Therefore, the input $16^{0} \mathrm{C}$ belongs by $98 \%$ to the FS $\mathrm{A}_{2}$, i.e. the fuzzy rule $\mathrm{A}_{2} \mathrm{x}$ $\mathrm{B}_{2}$ will be activated by $98 \%$. As a result, the corresponding triangle $\mathrm{B}_{2}$ in the output must be reduced to the 0.98 of its height (Fig. 9).

Also, in the triangle $\mathrm{HOZ}$ of Fig. 8 we have that $\frac{\mathrm{HO}}{\mathrm{IO}}=\frac{\mathrm{HZ}}{\mathrm{TZ}}$ and $\frac{\mathrm{ZH}}{\mathrm{ZT}}=\frac{\mathrm{ZO}}{\mathrm{ZE}}=\frac{18}{2}$, which gives that $\mathrm{IO}=\frac{1}{9}=\mathrm{TE}$. Then $(\mathrm{TEZ})=\frac{1}{9},(\mathrm{HOZ})=9$ and $\frac{(\mathrm{TEZ})}{(\mathrm{HOZ})}=\frac{1}{81} \square 0.012$. Therefore the fuzzy rule $\mathrm{A}_{1} \times \mathrm{B}_{1}$ will be activated by $1.2 \%$, which means that the corresponding triangle $\mathrm{B}_{1}$ in the output must be reduced to the 0.12 of its height (Fig. 9).

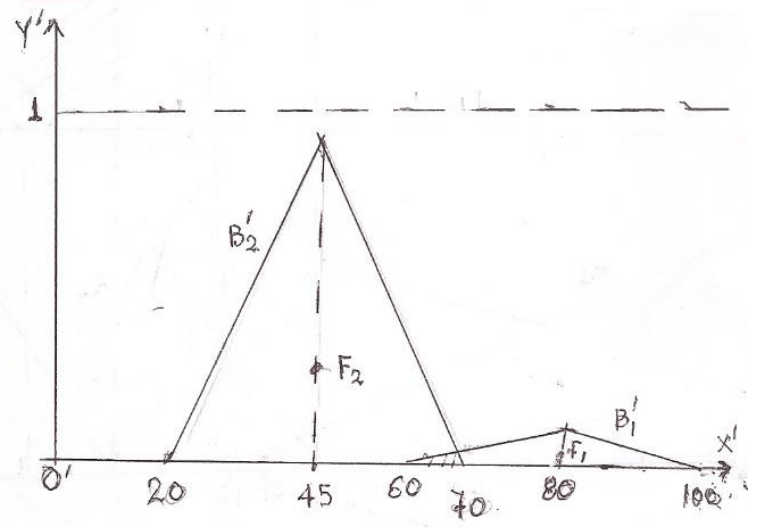

Fig. 9. The reduced triangles $\mathrm{B}_{1}$ ' and $\mathrm{B}_{2}$ ' (outputs) from Fig. 7

Let $F_{1}$ and $F_{2}$ be in Fig. 9 the COGs of the triangles $B_{1}$ ' and $\mathrm{B}_{2}$ ' with $\mathrm{x}$-coordinates $\mathrm{x}_{1}=80$ and $\mathrm{x}_{2}=45$ respectively. Then the $\mathrm{x}$-coordinate of the COG of the whole area defined by the two triangles, in which their common part is included twice (since it belongs to both outputs), is calculated by the equation

$$
\mathrm{x}=\frac{1}{\mathrm{~S}}\left(\mathrm{x}_{1} \mathrm{~S}_{1}+\mathrm{x}_{2} \mathrm{~S}_{2}\right)
$$

In equation (2) $\mathrm{S}_{\mathrm{i}}$ denotes the area of the triangle $\mathrm{B}_{\mathrm{i}}{ }^{\prime}, \mathrm{i}=1$, 2 and $\mathrm{S}=\mathrm{S}_{1}+\mathrm{S}_{2}$ (system of the COGs $\mathrm{F}_{1}$ and $\mathrm{F}_{2}$; e.g. see Wikipedia, 2014). But $\mathrm{S}_{1}=(0.12 \times 40): 2=2.4, \mathrm{~S}_{2}=(0.98 \times$ $50): 2=24.5$ and replacing those values to (2) one finds that $\mathrm{x} \approx 48$. Therefore, the rate of the boiler's operation increases and the temperature rises up again.

The process described above is performed by a fuzzy chip, which, receives a number from the input, compares it to all the input's FSs, provides output FSs and turns them to a number. Those steps are repeated continuously in millions of flips per second. That process is usually referred as Fuzzy Associative Memory (FAM). Note that computers have a direct memory, since they save an acquired information in an address - i.e. a set of digital characters determining a place in their memory - and, whenever is needed, they search for this information in that particular address. On the contrary, FAM activates the whole memory of the FCS in a way analogous to what humans do when they search all their past memory in order to recognize a familiar person among the crowd. Thus, FAM provides a reliable model of the way that humans think and decide. 


\section{Conclusions}

The discussion performed in this paper leads to the following general conclusions:

- FS theory and FL which is based on this theory, initiated by Zadeh approximately 50 years ago, although they have initially received tough critiques from several directions, they have enabled the scientists to model under conditions of imprecision and/or vagueness. FL has found nowadays many and important applications to almost all sectors of human activity, becoming a complement and support of probability theory, which is suitable for modelling only situations of uncertainty which are due to randomness.

- The traditional CSs are used to achieve, through automatic operation, increased productivity, high quality products and improved performance of a device or another system in terms of a set of bivalent IF - THEN rules. A model is needed in order to describe the operation of a traditional CS. The operation of the PID controllers, initiated by Minorsky in 1922, can be described by one or more differential equations that define the CS's response to its outputs.

- The operation of a FCS is based on the FAT theorem, according to which a system can be "covered" or approximated by a fuzzy system. An advantage of FCs with respect to the traditional CSs is that they do not need a model to describe their operation. On the contrary, they do need IF - THEN fuzzy rules, which, however, could not be too many as in a traditional controller. A FCS operates on the basis of its FAM, which simulates the way that humans think and decide. Starting from Japan during the 1980's, fuzzy controllers have found many industrial applications, and not only, while the research about them is nowadays in a phase of continuous development.

- The present work provides a basic framework, which is necessary for those willing to study deeper the operation of the fuzzy controllers and perhaps to apply its theoretical principles for the design of new "clever" constructions (industrial engines, home devices, etc.), as well as for the development of new "clever" approaches in automation, computer engineering, operation research, life sciences, sociology and in several other sectors of the human activity. In the effort of presenting the results of our study in the simplest possible way, so that they can be understood even by a non-expert, we chose a geometrical approach based on an example with a building's central heating controller.

\section{References}

[1] E. Athanassopoulos and M.Gr. Voskoglou, "A Philosophical Treatise on the Connection of Scientific Reasoning with Fuzzy Logic", Mathematics, vol. 8, article 875, 2020.

[2] M. Black, "Vagueness", Phil. of Science, Vol. 4, pp. 427-455, 1937. Reprinted in Language and Philosophy: Studies in Method, Cornell University Press, Ithaca and London, pp. 23-58, 1949. Also in Int. J. of General Systems, vol. 17, pp. 107-128, 1990.

[3] S. Iqbal (Ed.), Fuzzy Controllers: Recent Advances in Theory and Applications, IntechOpen: London, 2012

[4] A. Kaufmann and M. Gupta, Introduction to Fuzzy Arithmetic, Van Nostrand Reinhold Company: New York, 1991.

[5] B. Kosko, "Fuzziness Vs Probability", Int. J. of General Systems, vol. 17(2-3), pp. 211-240, 1990.

[6] B. Kosko, "Fuzzy Systems as Universal Approximators", in Proceedings of $1^{\text {st }}$ IEEE Conference on Fuzzy Systems, pp. 1153-1162, 1992.

[7] B. Kosko, Fuzzy Thinking: The New Science of Fuzzy Logic, Hyperion: New York, 1993.

[8] E.H. Mamdani and S. Assilian, "An experiment in linguistic synthesis with a fuzzy logic controller", Int. J. of Man-Machine Studies, vol. 7(1), pp. 1-13, 1975.

[9] K. Michels, F. Klawonn, R. Kruse, A. Nurnberger, Fuzzy Control: Fundamentals, Stability and Design, Springer: Berlin, 2006.

[10] Portal.dnb.de, GND, Katalog der Deutschen Nationalbibliothek (Authority control), https://portal.dnb.de/opac.htm?method=simple Search\&cqlMode=true\&query=nid=4032317-1, retrieved April 26, 2020.

[11] L. Reznik, Fuzzy Controllers, Newnes: Melbourne, 1997.

[12] M. Sugeno, Industrial applications of fuzzy control, Elsevier Science Pub. Co., 1985.

[13] H.A. Taha, Operations Research - An Introduction, Second Edition, Collier Macmillan: New York - London, 1967.

[14] K. Tanaka and H.O. Wang, Fuzzy Control Systems Design and Analysis: A Linear Matrix Inequality Approach, Wiley-Interscience New York:, 2001.

[15] M.Gr. Voskoglou, "Methods for Assessing Human-Machine Performance under Fuzzy Conditions", Mathematics, vol. 7, article 230, 2019.

[16] M.Gr. Voskoglou, and A.-B Salem, "Benefits and Limitations of the Artificial with Respect to the Traditional Learning of Mathematics", Mathematics, vol. 8, article 611, 2020.

[17] I.G. Umbers and P.J. King, "An analysis of human decision-making in cement kiln control and the implications for automation", Int. J. of Man-Mach. Stud., vol. 12, pp. 11-23, 1980.

[18] L.A. Zadeh, "Fuzzy Sets", Information and Control, vol. 8, pp. 338$353,1965$.

[19] L.A. Zadeh, "Outline of a new approach to the analysis of complex systems and decision processes", IEEE Trans. Syst. Man Cybern., vol. 3, pp. 28-44, 1973.

[20] Wikipedia, Center of mass: A system of particles, retrieved from http://en.wikipedia.org/wiki/Center_of_mass\#A_system_of_particles, October, 2014

\section{Creative Commons Attribution License 4.0 (Attribution 4.0 International, CC BY 4.0)}

This article is published under the terms of the Creative Commons Attribution License 4.0 https://creativecommons.org/licenses/by/4.0/deed.en_US 